\title{
Continuum limit results \\ from 2+1 flavor Domain Wall QCD
}

\section{Enno E. Scholz*}

on behalf of the RBC/UKQCD-Collaborations

Institute for Theoretical Physics, University of Regensburg, 93040 Regensburg, Germany

E-mail: enno.scholz@physik.uni-regensburg.de

\begin{abstract}
Quantum Chromodynamics with two mass degenerate light quark flavors and an additional single heavier quark flavor has been simulated on the lattice using the domain wall fermion formulation. These simulations cover a range of dynamical pion masses between 290 and $420 \mathrm{MeV}$ and were performed at two different values for the lattice spacing with $1 / a=1.73$ and $2.28 \mathrm{GeV}$ and a linear lattice extent of $2.8 \mathrm{fm}$. This talk will focus on the determination of the kaon bag parameter $B_{K}$ and the semi-leptonic kaon form factor $K_{l 3}$. The various methods for extrapolating these quantities to the limit of physical light quark mass will be evaluated.
\end{abstract}

35th International Conference of High Energy Physics

July 22-28, 2010

Paris, France

${ }^{*}$ Speaker. 
The good chiral properties of the domain wall fermion formulation allow to extract many quantities relevant for phenomenological applications from lattice QCD simulations. In this report we will focus on the kaon bag parameter $B_{K}$ and the semi-leptonic kaon form factor $K_{l 3}$ and also briefly comment on $K \rightarrow \pi \pi$ decays.

In continuing previous work [1] the RBC/UKQCD-Collaborations now simulated and analyzed lattice QCD with 2 mass-degenerate light quark flavors and one heavy quark flavor, i.e. a $2+1$ flavor simulation, at two different lattice scales using the domain wall fermion formulation. The light quark masses correspond to meson masses in the range of 290 to $420 \mathrm{MeV}$, whereas the heavier quark has been tuned to the mass of the strange quark as observed in Nature. Our coarser lattice spacing, where we simulated at two different values for the light quark masses, corresponds to a scale $1 / a=1.73(3) \mathrm{GeV}$, whereas on the finer lattice $(1 / a=2.28(3) \mathrm{GeV})$, three different light quark masses have been simulated. In the analysis also partially quenched quark masses were utilized, reaching down to meson masses of $225 \mathrm{MeV}$. The mass of the heavier strange quark was fixed separately on both sets of ensembles to reproduce its physical value in a first approximation up to $10-15$ per cent. Finally, we used the reweighting technique to fine tune the mass to its physical value. The physical, spatial volume in these simulations was held constant at $\approx(2.8 \mathrm{fm})^{3}$.

For the extrapolation to the physical light quark masses as realized in Nature we used two different ansätze: one based on $\mathrm{SU}_{L}(2) \times \mathrm{SU}_{R}(2)$ chiral perturbation theory (chPT) and as an alternative a simple first order Taylor expansion in the quark masses, cf. [1, 2, 3]. The scales of our simulations and the two physical quark masses $\left(m_{\mathrm{ud}}=\left(m_{\mathrm{u}}+m_{\mathrm{d}}\right) / 2\right.$ and $\left.m_{s}\right)$ were extracted using the masses of the pion, kaon, and the $\Omega^{-}$-baryon as input values.

\section{Neutral kaon mixing}

The mixing of of the neutral kaons due to indirect CP-violation is commonly described via the $\varepsilon_{K}$ parameter in the unitarity triangle analysis. Using the operator product expansion, it is possible to separate the QCD from the electro-weak contributions. The former have to be treated non-perturbatively, e.g. by measuring the kaon bag parameter, representing the relevant hadronic matrix element, in lattice QCD simulations. Previously, the RBC/UKQCD collaborations published results obtained with a single lattice spacing [ [4, 1]. Here we present our updated preliminary value, now also including the continuum extrapolation, for the kaon bag parameter [2]

$$
B_{K}^{\overline{\mathrm{MS}}}(\mu=2 \mathrm{GeV})=0.546(0.007)_{\mathrm{stat}+\mathrm{spread}}(0.016)_{\text {chiral extr }}(0.003)_{\mathrm{FV}}(0.014)_{\mathrm{ren}} .
$$

The extrapolation to the physical point (determined as described above) has been performed using either SU(2)-chPT (NLO) or an analytic ansatz based on a first order Taylor expansion, see Fig. 1. As our final value we take the average of these two ansätze and assign the difference as the systematic error due to the chiral extrapolation. The continuum limit is taken by including terms proportional to $a^{2}$ in $\mathrm{LO}$ in our extrapolation formulae and finally extrapolating to $a \rightarrow 0$. Finite volume (FV) corrections are included in the chPT fits. To quote our final result in the $\overline{\mathrm{MS}}$ scheme, the matrix element is first non-perturbatively renormalized in the RI/SMOM-scheme [5], which differs from the previously used RI/MOM scheme in that the momenta are defined symmetrically with non-exceptional external momenta. We found this advantageous in further reducing the systematic error from the renormalization procedure. The final conversion to the NDR-scheme 


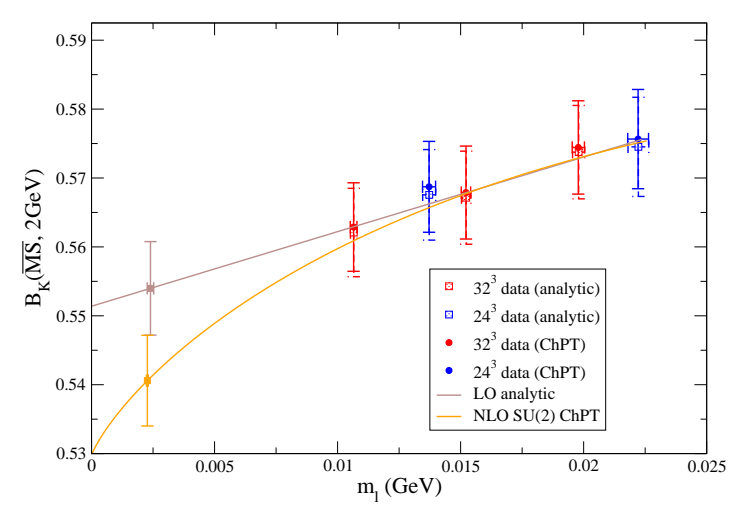

Figure 1: Extrapolation to the physical point for $B_{K}$ using either NLO SU(2) chPT or an analytic ansatz. Plot courtesy of C. Kelly [邶.

$(\overline{\mathrm{MS}})$ is performed using 1-loop perturbation theory. We indicate the systematic error from the renormalization procedure in our final result, and also enlarge the statistical error by the spread due to effects from $O(4)$-breaking seen in the extrapolation of the momenta in the non-perturbative renormalization. More details will be available in a forthcoming publication [6].

\section{Kaon semi-leptonic form factor}

The kaon semi-leptonic form factor $f_{+}^{K \pi}(0)$ in the $K_{l 3}$ mode of the $K \rightarrow \pi$ decay has been calculated on the Domain Wall QCD ensembles with the coarser lattice spacing [7], 8]. As is customary in lattice calculations of this quantity, the deviation $\Delta f=f_{+}^{K \pi}(0)-1-f_{2}\left(f_{0}, m_{\pi}, m_{K}\right)$ from unity in the $\mathrm{SU}(3)$-flavor limit and the analytic contribution $f_{2}$ is determined. Using spatial periodic boundary conditions, the discrete momenta accessible on the lattice need to be interpolated to zero momentum, which was done using a pole ansatz for $f_{+}^{K \pi}\left(q^{2}\right)$ in the earlier publication [7]. This introduces some unwanted model dependence in the final result. To overcome this shortcoming, partially twisted boundary conditions were introduced recently [9] allowing to tune the lattice momenta close to zero. This method has been successfully applied in the $K_{l 3}$ form factor calculation [8]. In the left panel of Fig. 2] the $q^{2}$-interpolation of the previous result is compared to the new data at $q^{2}$ close to zero, basically confirming the validity of the pole ansatz used previously. The right panel of Fig. 2 shows the chiral extrapolation to the physical point using SU(3) chPT resulting in [8]

$$
f_{+}^{K \pi}=0.9599(0.0034)_{\text {stat }}\left({ }_{-0.0043}^{+0.0031}\right)_{\text {chiralextr }}(0.0014)_{\text {discretization }} .
$$

The systematic error for the chiral extrapolation was estimated by allowing the pion decay constant in the $\mathrm{SU}(3)$ chiral limit, $f_{0}$, to vary in the range of $100-131 \mathrm{MeV}$. The discretization error takes into account the missing continuum extrapolation. The latter will be addressed in future work, when also data from the finer lattice spacing will be taken into account. Combining the above result for the form factor with the experimental result $\left|V_{u s} f_{+}(0)\right|=0.2163(5)$ [10] gives $V_{u s}=0.2253(14)$.

\section{3. $K \rightarrow \pi \pi$}

Calculating the amplitudes in the $K \rightarrow \pi \pi$ matrix elements via SU(3)-chPT from those in $K \rightarrow \pi$ and $K \rightarrow$ vacuum resulted in large systematic uncertainties [11] since the SU(3)-chPT for the simulated pion masses was not converging well. Recent approaches therefore focus on directly accessing the matrix elements via two-pion states on the available domain wall ensembles. For details we refer to [12, 13]. 

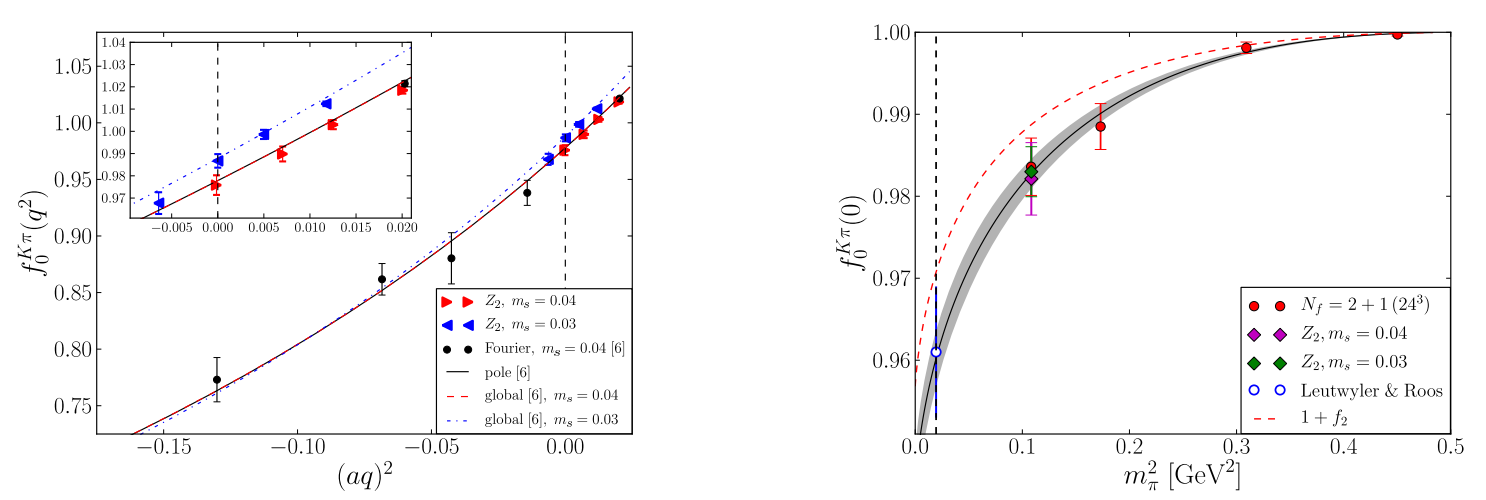

Figure 2: Left panel: interpolation in $q^{2}$ for $K_{l 3}$, right panel: extrapolation to physical light quark masses. Plots courtesy of P. Boyle et al. [8].

\section{Final remarks}

Selected results from the Domain Wall QCD research programme conducted by the RBC/UKQCD-Collaborations were presented with a special focus on kaon physics. Future simulations will aim at further reducing the simulated pion masses to reduce the current dominant systematic error from the extrapolation to the physical point. This will have to be achieved on coarser lattice spacings in order to keep the finite volume error well under control in numerically affordable simulations. For first results and how to still limit the discretization error on coarser lattices we refer to [14].

I am thankful to my colleagues from the RBC/UKQCD-Collaborations, especially P. Boyle, N. Christ, C. Kelly, Q. Liu, and C. Sachrajda. The speaker acknowledges support from the DFG SFB/TR 55 and E.U. REA grant PITN-GA-2009-238353 (ITN STRONGnet).

\section{References}

[1] RBC-UKQCD, C. Allton et al., Phys. Rev. D78, 114509 (2008), arXiv:0804.0473.

[2] RBC-UKQCD, C. Kelly, to be published in the proceedings of Lattice 2010 .

[3] RBC-UKQCD, Y. Aoki et al., (2010), arXiv:1011.0892.

[4] RBC-UKQCD, D. J. Antonio et al., Phys. Rev. Lett. 100, 032001 (2008), arXiv:hep-ph/0702042.

[5] C. Sturm et al., Phys. Rev. D80, 014501 (2009), arXiv:0901.2599.

[6] RBC-UKQCD, P. A. Boyle et al., in preparation .

[7] P. A. Boyle et al., Phys. Rev. Lett. 100, 141601 (2008), arXiv:0710.5136.

[8] P. A. Boyle et al., (2010), arXiv:1004.0886.

[9] P. A. Boyle, J. M. Flynn, A. Jüttner, C. T. Sachrajda, and J. M. Zanotti, JHEP 05, 016 (2007), arXiv:hep-lat/0703005.

[10] M. Antonelli et al., Eur. Phys. J. C69, 399 (2010), arXiv:1005.2323.

[11] S. Li and N. H. Christ, PoS LATTICE2008, 272 (2008), arXiv:0812.1368.

[12] RBC-UKQCD, E. Goode and M. Lightman, to be published in the proceedings of Lattice 2010 .

[13] RBC-UKQCD, Q. Liu, (2010), arXiv:1010.3768.

[14] RBC-UKQCD, R. D. Mawhinney, to be published in the proceedings of Lattice 2010 . 